\title{
Random intersection graphs with tunable degree distribution and clustering
}

\author{
Maria Deijfen* Willemien Kets ${ }^{\dagger}$
}

August 6, 2008

\begin{abstract}
A random intersection graph is constructed by assigning independently to each vertex a subset of a given set and drawing an edge between two vertices if and only if their respective subsets intersect. In this paper a model is developed in which each vertex is given a random weight, and vertices with larger weights are more likely to be assigned large subsets. The distribution of the degree of a given vertex is characterized and is shown to depend on the weight of the vertex. In particular, if the weight distribution is a power law, the degree distribution will be so as well. Furthermore, an asymptotic expression for the clustering in the graph is derived. By tuning the parameters of the model, it is possible to generate a graph with arbitrary clustering, expected degree and - in the power law case - tail exponent.
\end{abstract}

Keywords: Random intersection graphs, degree distribution, power law distribution, clustering, social networks.

AMS 2000 Subject Classification: 05C80, 91D30.

\section{Introduction}

During the last decade there has been a large interest in the study of large complex networks; see e.g. Dorogovtsev and Mendes (2003) and Newman et al. (2006) and the references therein. Due to the rapid increase in computer power, it has become possible to investigate various types of real networks such as social contact structures, telephone networks, power grids, the Internet and the World Wide Web. The empirical observations reveal that many of these networks have similar properties. For instance, they typically have power law degree sequences, that is, the fraction of vertices with degree $k$ is proportional to $k^{-\tau}$ for some exponent $\tau>1$. Furthermore, many networks are highly clustered, meaning roughly that there is a large number of triangles and other short cycles. In a social network, this is explained by the fact that two people who have a common friend often meet and become friends,

\footnotetext{
*Stockholm University. E-mail: mia@math.su.se

${ }^{\dagger}$ Santa Fe Institute and Tilburg University. E-mail: willemien.kets@santafe.edu
} 
creating a triangle in the network. A related explanation is that human populations are typically divided into various subgroups - working places, schools, associations etc - which gives rise to high clustering in the social network, since members of a given group typically know each other; see Palla et al. (2005) for some empirical observations.

Real-life networks are generally very large, implying that it is a time-consuming task to collect data to delineate their structure in detail. This makes it desirable to develop models that capture essential features of the real networks. A natural candidate to model a network is a random graph, and, to fit with the empirical observations, such a graph should have a heavy-tailed degree distribution and considerable clustering. We will quantify the clustering in a random graph by the conditional probability that three given vertices constitute a triangle, given that two of the three possible links between them exist. Other (empirical) definitions occur in the literature - see e.g. Newman (2003) - but they all capture essentially the same thing.

Obviously, the classical Erdös-Rényi graph will not do a good job as a network model, since the degrees are asymptotically Poisson distributed. Moreover, existing models for generating graphs with a given degree distribution - see e.g. Molloy and Reed (1995, 1998) - typically have zero clustering in the limit. In this paper, we propose a model, based on the so-called random intersection graph, where both the degree distribution and the clustering can be controlled. More precisely, the model makes it possible to obtain arbitrary prescribed values for the clustering and to control the mean and the tail behavior of the degree distribution.

\subsection{Description of the model}

The random intersection graph was introduced in Singer (1995) and Karoński et al. (1999), and has been further studied and generalized in Fill et al. (2000), Godehardt and Jaworski (2002), Stark (2004) and Jaworksi et al. (2006). Newman (2003) and Newman and Park (2003) discuss a similar model. In its simplest form the model is defined as follows.

1. Let $\mathcal{V}=\{1, \ldots, n\}$ be a set of $n$ vertices and $\mathcal{A}$ a set of $m$ elements. For $p \in[0,1]$, construct a bipartite graph $B(n, m, p)$ with vertex sets $\mathcal{V}$ and $\mathcal{A}$ by including each one of the $n m$ possible edges between vertices from $\mathcal{V}$ and elements from $\mathcal{A}$ independently with probability $p$.

2. The random intersection graph $G(n, m, p)$ with vertex set $\mathcal{V}$ is obtained by connecting two distinct vertices $i, j \in \mathcal{V}$ if and only if there is an element $a \in \mathcal{A}$ such that both $i$ and $j$ are adjacent to $a$ in $B(n, m, p)$.

When the vertices in $\mathcal{V}$ are thought of as individuals and the elements of $\mathcal{A}$ as social groups, this gives rise to a model for a social network in which two individuals are joined by an edge if they share at least one group. In the following, we frequently borrow the terminology from the field of social networks and refer to the vertices as individuals and the elements of $\mathcal{A}$ as groups, with the understanding that the model is of course much more general. 
To get an interesting structure, the number of groups $m$ is typically set to $m=$ $\left\lfloor n^{\alpha}\right\rfloor$ for some $\alpha>0$; see Karoński et al. (1999). We will assume this form for $m$ in the following. Let $D_{i}$ be the degree of vertex $i \in \mathcal{V}$ in $G(n, m, p)$. The probability that two individuals do not share a group in $B(n, m, p)$ is $\left(1-p^{2}\right)^{m}$. It follows that the edge probability in $G(n, m, p)$ is $1-\left(1-p^{2}\right)^{m}$ and hence the expected degree is

$$
\begin{aligned}
\mathbf{E}\left[D_{i}\right] & =(n-1)\left(1-\left(1-p^{2}\right)^{m}\right) \\
& =(n-1)\left(m p^{2}+O\left(m^{2} p^{4}\right)\right) .
\end{aligned}
$$

To keep the expected degree bounded as $n \rightarrow \infty$, we let $p=\gamma n^{-(1+\alpha) / 2}$ for some constant $\gamma>0$. We then have that $\mathbf{E}\left[D_{i}\right] \rightarrow \gamma^{2}$.

Stark (2004; Theorem 2) shows that in a random intersection graph with the above choice of $p$, the distribution of the degree of a given vertex converges to a point mass at 0 , a compound Poisson distribution or a Poisson distribution depending on whether $\alpha<1, \alpha=1$ or $\alpha>1$. This means that the current model cannot account for the power law degree distributions typically observed in real networks.

In the above formulation of the model, the number of groups that a given individual belongs to is binomially distributed with parameters $m$ and $p$. A generalization of the model, allowing for an arbitrary group distribution, is described in Godehardt and Jaworski (2002). The degree of a given vertex in such a graph is analyzed in Jaworski et al. (2006), where conditions on the group distribution are specified under which the degree is asymptotically Poisson distributed.

In the current paper, we are interested in obtaining graphs where non-Poissonian degree distributions can be identified. To this end, we propose a generalization of the original random intersection graph where the edge probability $p$ is random and depends on weights associated with the vertices. Other work in this spirit include for instance Chung and Lu (2002:1,2), Yao et al. (2005), Britton et al. (2006), Bollobás et al. (2007) and Deijfen et al. (2007). The model is defined as follows:

1. Let $n$ be a positive integer, and define $m=\left\lfloor\beta n^{\alpha}\right\rfloor$ with $\alpha, \beta>0$. As before, take $\mathcal{V}=\{1, \ldots, n\}$ to be a set of $n$ vertices and $\mathcal{A}$ a set of $m$ elements. Also, let $\left\{W_{i}\right\}$ be an i.i.d. sequence of positive random variables with distribution $F$, where $F$ is assumed to have mean 1 if the mean is finite. Finally, for some constant $\gamma>0$, set

$$
p_{i}=\gamma W_{i} n^{-(1+\alpha) / 2} \wedge 1 .
$$

Now construct a bipartite graph $B(n, m, F)$ with vertex sets $\mathcal{V}$ and $\mathcal{A}$ by adding edges to the elements of $\mathcal{A}$ for each vertex $i \in \mathcal{V}$ independently with probability $p_{i}$.

2. The random intersection graph $G(n, m, F)$ is obtained as before by drawing an edge between two distinct vertices $i, j \in \mathcal{V}$ if and only if they have a common adjacent vertex $a \in \mathcal{A}$ in $B(n, m, F)$.

In the social network setting, the weights can be interpreted as a measure of the social activity of the individuals. Indeed, vertices with large weights are more 
likely to join many groups and thereby acquire many social contacts. There are several other examples of real networks where the success of a vertex (measured by its degree) depends on some specific feature of the vertex; see e.g. Palla et al. (2005) for an example in the context of protein interaction networks. Furthermore, an advantage of the model is that it has an explicit and straightforward construction which, as we will see, makes it possible to exactly characterize the degree distribution and the clustering in the resulting graph.

\subsection{Results}

Our results concern the degree distribution and the clustering in the graph $G(n, m, F)$ as $n \rightarrow \infty$. More precisely, we will take the parameters $\alpha, \beta, \gamma$ and the weight distribution $F$ to be fixed (independent of $n$ ) and then analyze the degree of a given vertex and the clustering in the graph as $n \rightarrow \infty$. It turns out that the behavior of these quantities will be different in the three regimes $\alpha<1, \alpha=1$ and $\alpha>1$ respectively. The interesting case is $\alpha=1$, in the sense that this is when both the degree distribution and the clustering can be controlled. The cases $\alpha<1$ and $\alpha>1$ are included for completeness.

As for the degree, we begin by observing that, if $F$ has finite mean, then the asymptotic mean degree of vertex $i$, conditional on $W_{i}$, is given by $\beta \gamma^{2} W_{i}$ for all values of $\alpha$.

Proposition 1.1 Let $D_{i}$ be the degree of vertex $i \in \mathcal{V}$ in a random intersection graph $G(n, m, F)$ with $m=\left\lfloor\beta n^{\alpha}\right\rfloor$ and $p_{i}$ as in (1). If $F$ has finite mean, then, for all values of $\alpha>0$, we have that $\mathbf{E}\left[D_{i} \mid W_{i}\right] \rightarrow \beta \gamma^{2} W_{i}$ as $n \rightarrow \infty$.

Proof. We prove the claim for vertex $i=1$. Define

$$
W_{j}^{\prime}=W_{j} \cdot \mathbf{1}_{\left\{W_{j} \leq n^{1 / 4}\right\}} \quad \text { and } \quad W_{j}^{\prime \prime}=W_{j} \cdot \mathbf{1}_{\left\{W_{j}>n^{1 / 4}\right\}}
$$

and let $D^{\prime}$ and $D^{\prime \prime}$ denote the degree of vertex 1 when $\left\{W_{j}\right\}_{j \neq 1}$ are replaced by $\left\{W_{j}^{\prime}\right\}$ and $\left\{W_{j}^{\prime \prime}\right\}$ respectively, that is, $D^{\prime}$ is the number of neighbors of 1 with weight smaller than or equal to $n^{1 / 4}$ and $D^{\prime \prime}$ is the number of neighbors with weight larger than $n^{1 / 4}$. Write $p_{j}^{\prime}$ and $p_{j}^{\prime \prime}$ for the analog of (1) based on the truncated weights.

Now, conditional on the weights, the probability that there is an edge between 1 and $j$ is $1-\left(1-p_{1} p_{j}\right)^{m}$. To see that $\mathbf{E}\left[D^{\prime \prime}\right] \rightarrow 0$ as $n \rightarrow \infty$, we observe that

$$
1-\left(1-p_{i} p_{j}^{\prime \prime}\right)^{m} \leq m p_{1} p_{j}^{\prime \prime}=\beta \gamma W_{1} n^{(\alpha-1) / 2} p_{j}^{\prime \prime} .
$$

Summing the expectation of the right-hand side over $j \neq 1$, keeping $W_{1}$ fixed, gives (recall the truncation at 1 in (10)

$$
\mathbf{E}\left[D^{\prime \prime}\right] \leq \beta \gamma n^{(1+\alpha) / 2} \mathbf{E}\left[p_{k}^{\prime \prime}\right] \leq \beta \gamma\left(\gamma \mathbf{E}\left[W_{k}^{\prime \prime}\right]+n^{(1+\alpha) / 2} \mathbf{P}\left(\gamma W_{k} \geq n^{(1+\alpha) / 2}\right)\right),
$$

where both terms on the right hand side converge to 0 as $n \rightarrow \infty$ since $F$ has finite mean. As for $D^{\prime}$, we have

$$
1-\left(1-p_{1} p_{j}^{\prime}\right)^{m}=\beta \gamma^{2} W_{1} W_{j}^{\prime} n^{-1}+O\left(W_{1}^{2}\left(W_{j}^{\prime}\right)^{2} n^{-2}\right) .
$$


The sum over $j \neq 1$ of the expectation of the first term equals $\beta \gamma^{2} W_{1} \mathbf{E}\left[W_{k}^{\prime}\right]$, where $\mathbf{E}\left[W_{k}^{\prime}\right] \rightarrow \mathbf{E}\left[W_{k}\right]=1$ (since $F$ has finite mean) and the sum of the expectation of the second term converges to $0\left(\right.$ since $\left.\left(W_{j}^{\prime}\right)^{2} \leq n^{1 / 2}\right)$. Since $D_{0}=D^{\prime}+D^{\prime \prime}$, this proves the proposition.

The following theorem, which is a generalization of Theorem 2 in Stark (2004), gives a full characterization of the degree distribution for different values of $\alpha$.

Theorem 1.1 Consider the degree $D_{i}$ of vertex $i \in \mathcal{V}$ in a random intersection graph $G(n, m, F)$ with $m=\left\lfloor\beta n^{\alpha}\right\rfloor$ and $p_{i}$ as in (11), and assume that $F$ has finite mean.

(a) If $\alpha<1$, then $D_{i}$ converges in distribution to a point mass at 0 as $n \rightarrow \infty$.

(b) If $\alpha=1$, then $D_{i}$ converges in distribution to a sum of a Poisson $\left(\beta \gamma W_{i}\right)$ distributed number of Poisson $(\gamma)$ variables, where all variables are independent.

(c) If $\alpha>1$, then $D_{i}$ is asymptotically Poisson $\left(\beta \gamma^{2} W_{i}\right)$ distributed.

To understand Theorem 1.1, note that the expected number of groups that individual $i$ belongs to is roughly $\beta \gamma W_{i} n^{(\alpha-1) / 2}$. If $\alpha<1$ and $W_{i}$ has finite mean, this converges to 0 in probability, so that the degree distribution converges to a point mass at 0 , as stated in (a) (the group size however goes to infinity, explaining why the expected degree is still positive in the limit). For $\alpha=1$, the number of groups that individual $i$ is a member of is $\operatorname{Poisson}\left(\beta \gamma W_{i}\right)$ distributed as $n \rightarrow \infty$, and the number of other individuals in each of these groups is approximately Poisson $(\gamma)$ distributed, which explains (b). Finally, for $\alpha>1$, individual $i$ belongs to infinitely many groups as $n \rightarrow \infty$. This means that the edges indicators will be asymptotically independent, giving rise to the Poisson distribution specified in (c).

Moving on to the clustering, write $E_{i j}$ for the event that individuals $i, j \in \mathcal{V}$ have a common group in the bipartite graph $B(n, m, F)$ - that is, $E_{i j}$ is equivalent to the event that there is an edge between vertices $i$ and $j$ in $G(n, m, F)$ - and let $\overline{\mathbf{P}}_{n}$ be the probability measure of $B(n, m, F)$ conditional on the weights $\left\{W_{1}, \ldots, W_{n}\right\}$. For distinct vertices $i, j, k \in \mathcal{V}$, define

$$
\bar{c}_{i, j, k}^{(n)}=\overline{\mathbf{P}}_{n}\left(E_{i j} \mid E_{i k}, E_{j k}\right),
$$

that is, $\bar{c}_{i, j, k}^{(n)}$ is the edge probability between $i$ and $j$ in $G(n, m, F)$ given that they are both connected to $k$, conditional on the weights. To quantify the asymptotic clustering in the graph we will use

$$
c(G):=\lim _{n \rightarrow \infty} \mathbf{E}\left[\bar{c}_{i, j, k}^{(n)}\right],
$$

where the expectation is taken over the weights, that is, $c(G)$ is the limiting probability that three given vertices constitute a triangle conditional on that two of the three possible edges between them exist (the vertices are indistinguishable, so indeed $c(G)$ does not depend on the particular choice of $i, j$ and $k$ ). This should be closely 
related to the limiting quotient of the number of triangles and the number of triples with at least two edges present, which is one of the empirical measures of clustering that occur in the literature; see e.g. Newman (2003). Establishing this connection rigorously however requires additional arguments.

The asymptotic behavior of $\bar{c}_{i, j, k}^{(n)}$ is specified in the following theorem. By bounded convergence it follows that $c(G)$ is obtained as the mean of the in-probabilitylimits.

Theorem 1.2 Let $\{i, j, k\}$ be three distinct vertices in a random intersection graph $G(n, m, F)$ with $m=\left\lfloor\beta n^{\alpha}\right\rfloor$ and $p_{i}$ as in (11). If $F$ has finite mean, then

(a) $\bar{c}_{i, j, k}^{(n)} \rightarrow 1$ in probability for $\alpha<1$;

(b) $\bar{c}_{i, j, k}^{(n)} \rightarrow\left(1+\beta \gamma W_{k}\right)^{-1}$ in probability for $\alpha=1$;

(c) $\bar{c}_{i, j, k}^{(n)} \rightarrow 0$ in probability for $\alpha>1$.

To understand Theorem 1.2, assume that $i$ and $k$ share a group and that $j$ and $k$ share a group. The probability that $i$ and $j$ also have a common group then depends on the number of groups that the common neighbor $k$ belongs to. Indeed, the fewer groups $k$ belongs to, the more likely it is that $i$ and $j$ in fact share the same group with $k$. Recall that the expected number of groups that $k$ belongs to is roughly $\beta \gamma W_{k} n^{(\alpha-1) / 2}$. If $\alpha>1$, this goes to 0 as $n \rightarrow \infty$. Since it is then very unlikely that $k$ belongs to more than one group when $n$ is large, two given edges $\{i, k\}$ and $\{j, k\}$ are most likely generated by the same group, meaning that $i$ and $j$ are connected as well. On the other hand, if $\alpha>1$, the number of groups that $k$ belongs to is asymptotically infinite. Hence, that $i$ and $j$ each belong to one of these groups, does not automatically make it likely that they actually belong to the same group. If $\alpha=1$, individual $k$ belongs to $\beta \gamma W_{k}$ groups on average, explaining the expression in part (b) of the theorem.

From Theorem 1.2 it follows that, to get a nontrivial tunable clustering, we should choose $\alpha=1$. Indeed, then we have $c(G)=\mathbf{E}\left[\left(1+\beta \gamma W_{k}\right)^{-1}\right]$ and, for a given weight distribution $F$ (with finite mean), $c(G)$ can be varied between 0 and 1 by adjusting the parameters $\beta$ and $\gamma$. Furthermore, when $\alpha=1$, the degree distribution for a given vertex is asymptotically compound Poisson with the weight of the vertex as one of the parameters - see Theorem 1.1 (b) - and it is not hard to see that, if $F$ is a power law with exponent $\tau$, then the degree distribution will be so as well. Since the mean of $F$ is set to 1 , the expected asymptotic degree is $\beta \gamma^{2}$ by Proposition 1.1. Taken together, this means that, when $\alpha=1$, we can obtain a graph with a given value of the clustering and a power law degree distribution with prescribed exponent and prescribed mean by first choosing $F$ to be a power law with the desired exponent and then tuning the parameters $\beta$ and $\gamma$ to get the correct values of the clustering and the expected degree.

The rest of the paper is organized as follows. In Sections 2 and 3 , Theorem 1.1 and Theorem 1.2 are proved, respectively. The clustering is analyzed for the im- 
portant example of a power law weight distribution in Section 4. Finally, Section 5 provides an outline of possible future work.

\section{The degree distribution}

We begin by proving Theorem 1.1

Proof of Theorem 1.1. We prove the theorem for vertex $i=1$. Write $D_{1}=D$, and denote by $N$ the number of groups that individual 1 belongs to. Conditional on $W_{1}$, the variable $N$ is binomially distributed with parameters $m$ and $p_{1}$ and thus

$$
\overline{\mathbf{P}}_{n}(N=0)=\left(1-p_{1}\right)^{m} \geq 1-m p_{1} \geq 1-\beta \gamma^{2} W_{1}^{(\alpha-1) / 2} .
$$

For $\alpha<1$, the expectation of the last term converges to 0 as $n \rightarrow \infty$, and it follows from bounded convergence that $\mathbf{P}(N=0)=\mathbf{E}\left[\overline{\mathbf{P}}_{n}(N=0)\right] \rightarrow 1$. This proves (a), since clearly $D=0$ if individual 1 is not a member of any group.

To prove (b) and (c), first recall the definition of the weights $\left\{W_{i}^{\prime}\right\}$ and $\left\{W_{i}^{\prime \prime}\right\}-$ truncated from above and below respectively at $n^{1 / 4}$ - and the corresponding degree variables $D^{\prime}$ and $D^{\prime \prime}$ from the proof of Proposition 1.1. We have already showed (in proving Proposition 1.1) that $\mathbf{E}\left[D^{\prime \prime}\right] \rightarrow 0$, which implies that $D^{\prime \prime}$ converges to 0 in probability (indeed $\left.P\left(D^{\prime \prime}>0\right) \leq \mathbf{E}\left[D^{\prime \prime}\right]\right)$. Hence it suffices to show that the generating function of $D^{\prime}$ converges to the generating function of the claimed limiting distribution. To this end, we condition on the weight $W_{1}$, which is thus assumed to be fixed in what follows, and let $X_{i}^{\prime}(i=2, \ldots, n)$ denote the number of common groups of individual 1 and individual $i$ when the truncated weights $W_{i}^{\prime}$ are used for $i \neq 1$. Since two individuals are connected if and only if they have at least one group in common, we can write $D^{\prime}=\sum_{i=2}^{n} \mathbf{1}_{\left\{X_{i}^{\prime} \geq 1\right\}}$. Furthermore, conditional on $N$ and $\left\{W_{i}^{\prime}\right\}_{i \geq 2}$, the random variables $X_{i}^{\prime}, i=2, \ldots, n$, are independent and binomially distributed with parameters $N$ and $p_{i}^{\prime}=\gamma W_{i}^{\prime} n^{-(1+\alpha) / 2}$. Hence, with $\overline{\mathbf{P}}_{n}$ denoting the probability measure of the bipartite graph $B(n, m, F)$ conditional on both $\left\{W_{i}^{\prime}\right\}_{i \geq 2}$ and $N$, the generating function of $D^{\prime}$ can be written as

$$
\mathbf{E}\left[t^{D^{\prime}}\right]=\mathbf{E}\left[\prod_{i=2}^{n} \mathbf{E}\left[t^{\mathbf{1}\left\{X_{i}^{\prime} \geq 1\right\}} \mid\left\{W_{i}^{\prime}\right\}, N\right]\right]=\mathbf{E}\left[\prod_{i=2}^{n}\left(1+(t-1) \overline{\overline{\mathbf{P}}}_{n}\left(X_{i}^{\prime} \geq 1\right)\right)\right]
$$

where $t \in[0,1]$. Using the Taylor expansion $\log (1+x)=x+O\left(x^{2}\right)$ and the fact that

$$
\overline{\overline{\mathbf{P}}}_{n}\left(X_{i}^{\prime} \geq 1\right)=1-\left(1-p_{i}^{\prime}\right)^{N}=N p_{i}^{\prime}+O\left(N^{2}\left(p_{i}^{\prime}\right)^{2}\right)
$$

we get that

$$
\prod_{i=2}^{n}\left(1+(t-1) \overline{\overline{\mathbf{P}}}_{n}\left(X_{i}^{\prime} \geq 1\right)\right)=e^{(t-1) N \sum p_{i}^{\prime}+O\left(N^{2} \sum\left(p_{i}^{\prime}\right)^{2}\right)} .
$$

Defining

$$
R_{n}:=\prod_{i=2}^{n}\left(1+(t-1) \overline{\overline{\mathbf{P}}}_{n}\left(X_{i}^{\prime} \geq 1\right)\right)-e^{(t-1) N \sum p_{i}^{\prime}}
$$


we therefore have that

$$
R_{n}=e^{(t-1) N \sum p_{i}}\left(e^{O\left(N^{2} \sum\left(p_{i}^{\prime}\right)^{2}\right)}-1\right) .
$$

Since the product in (3) is the conditional expectation of $t^{D^{\prime}}$ with $t \in[0,1]$, it takes values between 0 and 1 and, since $e^{(t-1) N \sum p_{i}} \in(0,1]$, it follows that $R_{n} \in[-1,1]$. Furthermore, recalling that $W_{i}^{\prime} \leq n^{1 / 4}$, we have for $\alpha \geq 1$ that

$$
N^{2} \sum_{i=2}^{n}\left(p_{i}^{\prime}\right)^{2}=N^{2} \gamma^{2} n^{-(1+\alpha)} \sum_{i=2}^{n}\left(W_{i}^{\prime}\right)^{2} \leq N^{2} \gamma^{2} n^{-1 / 2},
$$

implying that $R_{n} \rightarrow 0$ in probability and thus, by bounded convergence, $\mathbf{E}\left[R_{n}\right] \rightarrow 0$. Hence we are done if we show that

(i) $\mathbf{E}\left[e^{(t-1) N \sum p_{i}}\right] \rightarrow e^{\beta \gamma W_{1}\left(e^{\gamma(t-1)}-1\right)}$ if $\alpha=1$;

(ii) $\mathbf{E}\left[e^{(t-1) N \sum p_{i}}\right] \rightarrow e^{\beta \gamma^{2} W_{1}(t-1)}$ if $\alpha>1$,

where the limits are recognized as the generating functions for the desired compound Poisson and Poisson distribution in part (b) and (c) of the theorem, respectively. To this end, note that the expectation with respect to $N$ of $e^{(t-1) N \sum p_{i}^{\prime}}$ is given by the generating function for $N$ evaluated at the point $e^{(t-1) \sum p_{i}^{\prime}}$. Since $N$ is binomially distributed with parameters $m$ and $p_{1}$, we have that

$$
\mathbf{E}\left[e^{(t-1) N \sum p_{i}^{\prime}}\right]=\mathbf{E}\left[\left(1+p_{1}\left(e^{(t-1) \sum p_{i}^{\prime}}-1\right)\right)^{m}\right] .
$$

For $\alpha=1$, we have $m=\lfloor\beta n\rfloor$ and $p_{i}^{\prime}=\gamma W_{i}^{\prime} n^{-1}$. Recalling that $\mathbf{E}\left[W_{i}^{\prime}\right] \rightarrow \mathbf{E}\left[W_{i}\right]=1$, it follows that $\sum p_{i}^{\prime} \rightarrow \gamma$ almost surely. Hence,

$$
\left(1+p_{1}\left(e^{(t-1) \sum p_{i}^{\prime}}-1\right)\right)^{\lfloor\beta n\rfloor} \rightarrow e^{\beta \gamma W_{1}\left(e^{\gamma(t-1)}-1\right)} \text { a.s. as } n \rightarrow \infty,
$$

and it follows from bounded convergence that the expectation converges to the same limit, proving (i).

For $\alpha>1$, define $\tilde{p}_{i}^{\prime}=n^{(\alpha-1) / 2} p_{i}^{\prime}$. With $m=\left\lfloor\beta n^{\alpha}\right\rfloor$ and $p_{1}=\gamma W_{1} n^{-(1+\alpha) / 2} \wedge 1$, we get after some rewriting, that

$$
\left(1+p_{1}\left(e^{(t-1) \sum p_{i}^{\prime}}-1\right)\right)^{m}=\left(1+\frac{\gamma W_{1}(t-1) \sum \tilde{p}_{i}^{\prime}}{n^{\alpha}} \cdot \frac{e^{(t-1) n^{(1-\alpha) / 2} \sum \tilde{p}_{i}^{\prime}-1}}{(t-1) n^{(1-\alpha) / 2} \sum \tilde{p}_{i}^{\prime}}\right)^{\left\lfloor\beta n^{\alpha}\right\rfloor}
$$

By the law of large numbers, $\sum \tilde{p}_{i}^{\prime} \rightarrow \gamma$ almost surely, and, since $\left(e^{x}-1\right) / x \rightarrow 1$ as $x \rightarrow 0$, it follows that the right hand side above converges to $e^{\beta \gamma^{2} W_{1}(t-1)}$ almost surely as $n \rightarrow \infty$. By (4) and bounded convergence, this proves (ii). 


\section{Clustering}

In this section, we prove Theorem 1.2. First recall that $E_{i j}$ denotes the event that the individuals $i, j \in \mathcal{V}$ share at least one group. It will be convenient to extend this notation. To this end, for $i, j, k \in \mathcal{V}$, denote by $E_{i j k}$ the event that there is at least one group to which all three individuals $i, j$ and $k$ belong, and write $E_{i j, i k, j k}$ for the event that there are at least three distinct groups to which $i$ and $j, i$ and $k$, and $j$ and $k$ respectively belong. Similarly, the event that there are two distinct groups to which individuals $i$ and $k$, and $j$ and $k$ respectively belong is denoted by $E_{i k, j k}$. The proof of Theorem 1.2 relies on the following lemma.

Lemma 3.1 Consider a random intersection graph $G(n, m, F)$ with $m=\left\lfloor\beta n^{\alpha}\right\rfloor$ and $p_{i}$ defined as in (1). For any three distinct vertices $i, j, k \in \mathcal{V}$, we have that

(a) $\overline{\mathbf{P}}_{n}\left(E_{i j k}\right)=\frac{\beta \gamma^{3} W_{i} W_{j} W_{k}}{n^{(3+\alpha) / 2}}+O\left(\frac{W_{i}^{2} W_{j}^{2} W_{k}^{2}}{n^{3+\alpha}}\right)$;

(b) $\overline{\mathbf{P}}_{n}\left(E_{i j, i k, j k}\right)=\frac{\beta^{3} \gamma^{6} W_{i}^{2} W_{j}^{2} W_{k}^{2}}{n^{3}}+O\left(\frac{W_{i}^{3} W_{j}^{3} W_{k}^{3}}{n^{4}}\right)$;

(c) $\overline{\mathbf{P}}_{n}\left(E_{i k, j k}\right)=\frac{\beta^{2} \gamma^{4} W_{i} W_{j} W_{k}^{2}}{n^{2}}+O\left(\frac{W_{i}^{2} W_{j}^{2} W_{k}^{3}}{n^{3}}\right)$;

(d) $\overline{\mathbf{P}}_{n}\left(E_{i j k} E_{i k, j k}\right)=O\left(\frac{W_{i}^{2} W_{j}^{2} W_{k}^{2}}{n^{(5+\alpha) / 2}}\right)$.

Proof. As for (a), the probability that three given individuals $i, j$ and $k$ do not share any group at all is $\left(1-p_{i} p_{j} p_{k}\right)^{m}$. Using the definitions of $m$ and the edge probabilities $\left\{p_{i}\right\}$, it follows that

$$
\overline{\mathbf{P}}_{n}\left(E_{i j k}\right)=1-\left(1-p_{i} p_{j} p_{k}\right)^{m}=\frac{\beta \gamma^{3} W_{i} W_{j} W_{k}}{n^{(3+\alpha) / 2}}+O\left(\frac{W_{i}^{2} W_{j}^{2} W_{k}^{2}}{n^{3+\alpha}}\right) .
$$

To prove (b), note that the probability that there is exactly one group to which both $i$ and $j$ belong is $m p_{i} p_{j}\left(1-p_{i} p_{j}\right)^{m-1}=m p_{i} p_{j}+O\left(m^{2} p_{i}^{2} p_{j}^{2}\right)$. Given that $i$ and $j$ share one group, the probability that $i$ and $k$ share exactly one of the other $m-1$ groups is $(m-1) p_{i} p_{k}\left(1-p_{i} p_{k}\right)^{m-2}=m p_{i} p_{k}+O\left(m^{2} p_{i}^{2} p_{k}^{2}\right)$. Finally, the conditional probability that there is a third group to which both $j$ and $k$ belong given that the pairs $i, j$ and $i, k$ share one group each is $1-\left(1-p_{j} p_{k}\right)^{m-2}=m p_{j} p_{k}+O\left(m^{2} p_{j}^{2} p_{k}^{2}\right)$. Combining these estimates, and noting that scenarios in which $i$ and $j$ or $i$ and $k$ share more than one group have negligible probability in comparison, we get that

$$
\begin{aligned}
\overline{\mathbf{P}}_{n}\left(E_{i j, i k, j k}\right) & =m^{3} p_{i}^{2} p_{j}^{2} p_{k}^{2}+O\left(m^{4} p_{i}^{2} p_{j}^{2} p_{k}^{2}\left(p_{i} p_{j}+p_{i} p_{k}+p_{j} p_{k}\right)\right) \\
& =\frac{\beta^{3} \gamma^{6} W_{i}^{2} W_{j}^{2} W_{k}^{2}}{n^{3}}+O\left(\frac{W_{i}^{3} W_{j}^{3} W_{k}^{3}}{n^{4}}\right) .
\end{aligned}
$$

Part (c) is derived analogously. 
As for (d), note that the event $E_{i j k} E_{i k, j k}$ occurs when there is at least one group that is shared by all three vertices $i, j$ and $k$ and a second group shared by either $i$ and $k$ or $j$ and $k$. Denote by $r$ the probability that individual $k$ and at least one of the individuals $i$ and $j$ belong to a fixed group. Then $r=p_{k}\left(p_{i}+p_{j}-p_{i} p_{j}\right)$, and, conditional on that there is exactly one group to which all three individuals $i$, $j$ and $k$ belong (the probability of this is $m p_{i} p_{j} p_{k}\left(1-p_{i} p_{j} p_{k}\right)^{m-1}=O\left(m p_{i} p_{j} p_{k}\right)$ ), the probability that there is at least one other group that is shared either by $i$ and $k$ or by $j$ and $k$ is $1-(1-r)^{m-1}=O(m r)$. It follows that

$$
\overline{\mathbf{P}}_{n}\left(E_{i j k} E_{i k, j k}\right)=O\left(m^{2} p_{i} p_{j} p_{k} r\right)=O\left(\frac{W_{i}^{2} W_{j}^{2} W_{k}^{2}}{n^{(5+\alpha) / 2}}\right) .
$$

Using Lemma 3.1, it is not hard to prove Theorem 1.2.

Proof of Theorem 1.2. Recall the definition (2) of $\bar{c}_{i, j, k}^{(n)}$ and note that

$$
\overline{\mathbf{P}}_{n}\left(E_{i j} \mid E_{i k} E_{j k}\right)=\frac{\overline{\mathbf{P}}_{n}\left(E_{i j k} \cup E_{i j, i k, j k}\right)}{\overline{\mathbf{P}}_{n}\left(E_{i j k} \cup E_{i k, j k}\right)} .
$$

As for (a), applying the estimates of Lemma 3.1 and merging the error terms yields

$$
\begin{aligned}
\overline{\mathbf{P}}_{n}\left(E_{i j} \mid E_{i k} E_{j k}\right) & \geq \frac{\overline{\mathbf{P}}_{n}\left(E_{i j k}\right)}{\overline{\mathbf{P}}_{n}\left(E_{i j k}\right)+\overline{\mathbf{P}}_{n}\left(E_{i k, j k}\right)} \\
& =\frac{1+O\left(W_{i} W_{j} W_{k} n^{-(3+\alpha) / 2}\right)}{1+W_{k}\left[\beta \gamma n^{(\alpha-1) / 2}+O\left(W_{i} W_{j} W_{k} n^{-(3-\alpha) / 2}\right)\right]}
\end{aligned}
$$

By Markov's inequality and the fact that $W_{i}, W_{j}$ and $W_{k}$ are independent and have finite mean, it follows that $W_{i} W_{j} W_{k} n^{-(3-\alpha) / 2}$ goes to 0 in probability when $\alpha<1$. Similarly, $W_{i} W_{j} W_{k} n^{-(3+\alpha) / 2} \rightarrow 0$ in probability. Hence, the quotient in (5) converges to 1 in probability for $\alpha<1$, as claimed.

To prove part (b), note that, for $\alpha=1$, the lower bound (5) for $\bar{c}_{i, j, k}^{(n)}$ converges in probability to $\left(1+\beta \gamma W_{k}\right)^{-1}$. To obtain an upper bound, we apply Lemma 3.1 with $\alpha=1$ to get that

$$
\begin{aligned}
\overline{\mathbf{P}}_{n}\left(E_{i j} \mid E_{i k} E_{j k}\right) & \leq \frac{\overline{\mathbf{P}}_{n}\left(E_{i j k}\right)+\overline{\mathbf{P}}_{n}\left(E_{i j, i k, j k}\right)}{\overline{\mathbf{P}}_{n}\left(E_{i j k}\right)+\overline{\mathbf{P}}_{n}\left(E_{i k, j k}\right)-\overline{\mathbf{P}}_{n}\left(E_{i j k} E_{i k, j k}\right)} \\
& =\frac{1+O\left(W_{i} W_{j} W_{k} n^{-1}\right)}{1+W_{k}\left[\beta \gamma+O\left(W_{i} W_{j} W_{k} n^{-1}\right)\right]} .
\end{aligned}
$$

Here $W_{i} W_{j} W_{k} n^{-1}$ converges to 0 in probability by Markov's inequaliy, and (b) follows.

As for (c), combining the bound in (6) with the estimates in Lemma 3.1 yields

$$
\overline{\mathbf{P}}_{n}\left(E_{i j} \mid E_{i k} E_{j k}\right) \leq \frac{n^{(1-\alpha) / 2}+O\left(W_{i} W_{j} W_{k} n^{-1}\right)}{n^{(1-\alpha) / 2}+W_{k}\left[\beta \gamma+O\left(W_{i} W_{j} W_{k} n^{-1}\right)\right]} .
$$

Since $W_{i} W_{j} W_{k} n^{-1} \rightarrow 0$ in probability, this bound converges to 0 in probability for $\alpha>1$, as desired. 


\section{Clustering for a power law weight distribution}

When $\alpha=1$, the clustering is given by $c(G)=\mathbf{E}\left[\left(1+\beta \gamma W_{k}\right)^{-1}\right]$. Here we investigate this expression in more detail for the important case that $F$ is a power law. More precisely, we take $F$ to be a Pareto distribution with density

$$
f(x)=\frac{(\lambda-2)^{\lambda-1}}{(\lambda-1)^{\lambda-2}} x^{-\lambda} \quad \text { for } x \geq \frac{\lambda-2}{\lambda-1} .
$$

When $\lambda>2$, this distribution has mean 1 , as desired. The asymptotic clustering $c(G)$ is given by the integral

$$
\frac{(\lambda-2)^{\lambda-1}}{(\lambda-1)^{\lambda-2}} \int_{\frac{\lambda-2}{\lambda-1}}^{\infty}(1+\beta \gamma x)^{-1} x^{-\lambda} d x
$$

Defining $u:=(\lambda-2) /(x \cdot(\lambda-1))$, we obtain

$$
\begin{aligned}
c(G) & =\frac{1}{\beta \gamma} \frac{(\lambda-1)^{2}}{(\lambda-2)} \int_{0}^{1} u^{\lambda-1}\left(1+\frac{u}{\beta \gamma}\left(\frac{\lambda-1}{\lambda-2}\right)\right)^{-1} d u \\
& =: \frac{1}{\beta \gamma \lambda} \frac{(\lambda-1)^{2}}{(\lambda-2)}{ }_{2} F_{1}\left(1, \lambda ; 1+\lambda ;-\frac{1}{\beta \gamma}\left(\frac{\lambda-1}{\lambda-2}\right)\right),
\end{aligned}
$$

where ${ }_{2} F_{1}$ is the hypergeometric function. For $\beta \gamma \geq(\lambda-1) /(\lambda-2)$, a series expansion of the integrand yields that

$$
\begin{aligned}
c(G) & =\frac{1}{\beta \gamma} \frac{(\lambda-1)^{2}}{(\lambda-2)} \sum_{k=0}^{\infty}\left(-\frac{1}{\beta \gamma}\left(\frac{\lambda-1}{\lambda-2}\right)\right)^{k} \frac{1}{k+\lambda} \\
& =: \frac{1}{\beta \gamma} \frac{(\lambda-1)^{2}}{(\lambda-2)} \Phi\left(-\frac{1}{\beta \gamma}\left(\frac{\lambda-1}{\lambda-2}\right), 1, \lambda\right),
\end{aligned}
$$

where $\Phi$ is the Lerch transcedent. Furthermore, when $\lambda$ is an integer, we get

$c(G)=\frac{(\lambda-2)^{\lambda-1}}{(\lambda-1)^{\lambda-2}}\left[(-\beta \gamma)^{\lambda-1} \ln \left(1+\frac{\lambda-1}{\beta \gamma(\lambda-2)}\right)+\sum_{\ell=1}^{\lambda-1} \frac{(-\beta \gamma)^{\lambda-1-\ell}}{\ell}\left(\frac{\lambda-1}{\lambda-2}\right)^{\ell}\right]$.

Figure 1 (a) and (b) show how the clustering depends on $\lambda$ and $\beta \gamma$ respectively. For any $c \in(0,1)$ and a given tail exponent $\lambda$, we can find a value of $\beta \gamma$ such that the clustering is equal to $c$. Combining this with a condition on $\beta \gamma^{2}$, induced by fixing the mean degree in the graph, the parameters $\beta$ and $\gamma$ can be specified.

\section{Future work}

Apart from the degree distribution and the clustering, an important feature of real networks is that there is typically significant correlation for the degrees of neighboring nodes, that is, either high (low) degree vertices tend to be connected to other vertices with high (low) degree (positive correlation), or high (low) degree vertices 
tend to be connected to low (high) degree vertices (negative correlation). A next step is thus to quantify the degree correlations in the current model. The fact that individuals share groups should indeed induce positive degree correlation, which agrees with empirical observations from social networks; see Newman (2003) and Newman and Park (2003).

Also other features of the model are worth investigating. For instance, many real networks are "small worlds", meaning roughly that the distances between vertices remain small also in very large networks. It would be interesting to study the relation between the distances between vertices, the degree distribution and the clustering in the current model.

Finally, dynamic processes behave differently on clustered networks as compared to more tree-like networks. Most work to date has focused on the latter class. In Britton et al. (2008) however, epidemics on random intersection graphs without random weights are studied and it is investigated how the epidemic spread is affected by the clustering in the graph. It would be interesting to extend this work to incorporate weights on the vertices, allowing to tune also the (tail of the) degree distribution and study its impact on the epidemic process.

Acknowledgement. We thank Remco van der Hofstad and Wouter Kager for valuable suggestions that have improved the manuscript.

\section{References}

Bollobás, B., Janson, S. and Riordan, O. (2007): The phase transition in inhomogeneous random graphs, Random Structures \& Algorithms 31, 3-122.

Britton, T., Deijfen, M. and Martin-Löf, A. (2006): Generating simple random graphs with prescribed degree distribution, Journal of Statistical Physics 124, 13771397.

Britton, T., Deijfen, M., Lagerås, A. and Lindholm, M. (2008): Epidemics on random graphs with tunable clustering, Journal of Applied Probability 45, 743-756.

Chung, F. and Lu. L (2002:1): Connected components in random graphs with given degree sequences, Annals of Combinatorics 6, 125-145.

Chung, F. and Lu. L (2002:2): The average distances in random graphs with given expected degrees, Proceedings of the National Academy of Sciences 99, 15879-15882.

Deijfen, M., vd Esker, H., vd Hofstad, R. and Hooghiemstra, G. (2007): A preferential attachment model with random initial degrees, Arkiv för Matematik, to appear.

Dorogovtsev, S. and Mendes, J. (2003): Evolution of Networks, from Biological Nets to the Internet and $W W W$, Oxford University Press.

Fill, J., Scheinerman, E. and Singer-Cohen, K. (2000): Random intersection graphs when $m=\omega(n)$ : an equivalence theorem relating the evolution of the $G(n, m, p)$ and $G(n, p)$ models, Random Structures \& Algorithms 16, 156-176. 
Godehardt, E. and Jaworski, J. (2002): Two models of random intersection graphs for classification, in Exploratory data analysis in empirical research, eds. Schwaiger M. and Opitz, O., Springer, 67-81.

Jaworski, J., Karoński, M. and Stark, D. (2006): The degree of a typical vertex in generalized random intersection graph models, Discrete Mathematics 306, 21522165 .

Karoński, M., Scheinerman, E. and Singer-Cohen, K. (1999): On random intersection graphs: the subgraphs problem, Combinatorics, Probability \& Computing 8, 131-159.

Molloy, M. and Reed, B. (1995): A critical point for random graphs with a given degree sequence, Random Structures \& Algorithms 6, 161179.

Molloy, M. and Reed, B. (1998): The size of the giant component of a random graph with a given degree sequence, Combinatorics, Probability \& Computing 7, 295-305.

Newman, M. E. J., Strogatz, S. H., and Watts, D. J. (2002): Random graphs with arbitrary degree distributions and their applications, Physical Review E 64, 026118.

Newman, M. E. J. (2003): Properties of highly clustered networks, Physical Review E 68, 026121.

Newman, M. E. J. and Park J. (2003): Why social networks are different from other types of networks, Physical Review E 68, 036122.

Palla, G., and Derényi, I., Farkas, I. and Vicsek, T. (2005): Uncovering the overlapping community structure of complex networks in nature and society, Nature $\mathbf{4 3 5}$, $814-818$.

Singer, K. (1995): Random intersection graphs, PhD thesis, Johns Hopkins University.

Stark, D. (2004): The vertex degree distribution of random intersection graphs, Random Structures \& Algorithms 24, 249-258.

Yao, X., Zhang, C., Chen, J. and Li, Y. (2005): On the scale-free intersection graphs, Lecture notes in computer science 3481, 1217-1224. 


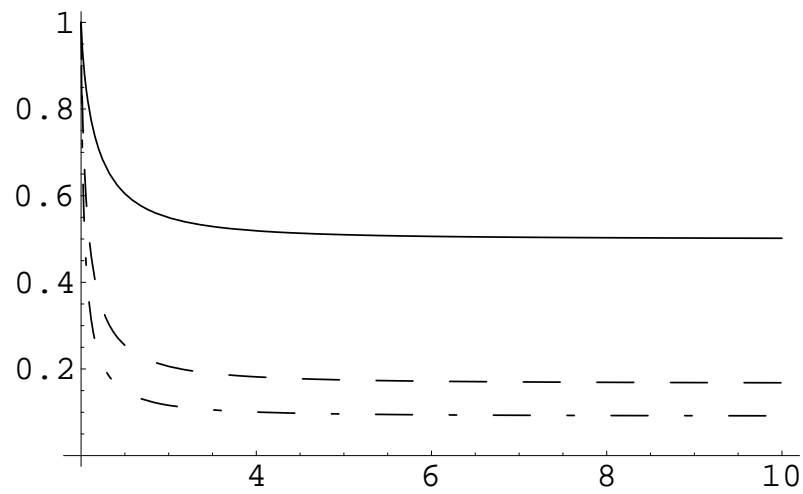

(a) The clustering as a function of $\lambda$ for different values of $\beta \gamma$ : $\beta \gamma=1(-), \beta \gamma=5(---), \beta \gamma=10(-\cdot-)$.

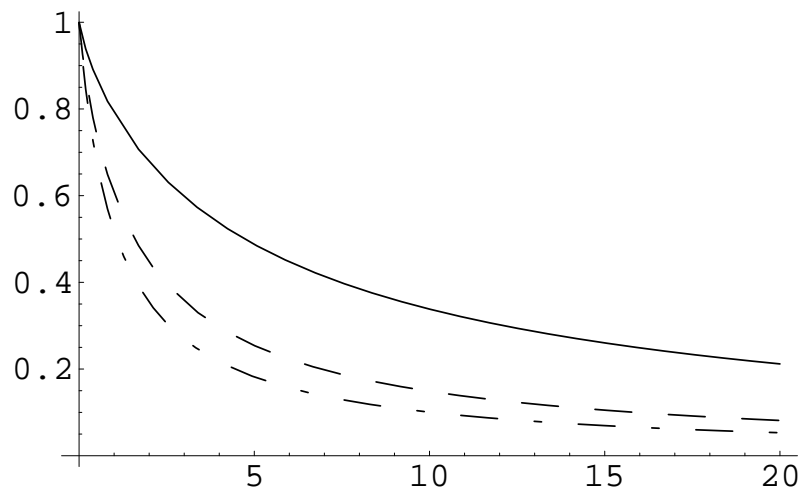

(b) The clustering as a function of $\beta \gamma$ for different values of $\lambda$ : $\lambda=2.1(-), \lambda=2.5(---), \lambda=4(-\cdot-)$.

Figure 1: Clustering for a power law distribution. 